\title{
A MOBILE ENVIRONMENTAL MONITORING STATION FOR SUSTAINABLE CITIES
}

\author{
L.T. SILVA, B. MENDES, D. RODRIGUES, P. RIBEIRO \& J.F.G. MENDES \\ CTAC- Centre of Territory, Environment and Construction, University of Minho, Campus de Gualtar, \\ 4710-057, Braga, Portugal.
}

\begin{abstract}
The aim of this paper is to present a mobile environmental monitoring station to evaluate the urban environment.The different phases of the mobile unit development are presented from its construction and operation mode for handling and sharing the data obtained. This mobile unit measures the following urban environmental parameters: environmental noise $\left(\mathrm{L}_{\mathrm{eq}}, \mathrm{L}_{10}\right.$ and $\left.\mathrm{L}_{90}\right)$, air quality $\left(\mathrm{PM}_{10}, \mathrm{PM}_{2.5}\right.$ and Total Suspended Particles) and meteorological parameters (air temperature, relative humidity, wind speed and wind direction).

This mobile unit is part of a project developed for the Portuguese city of Vila Real, called SMMART (in English "Municipal System for Monitoring Air, Noise and Traffic"). The municipality accepted the challenge of developing a platform whereby the population could be informed about the quality of the urban environment (air and noise) of the city where they live.

Keywords: air quality, environmental noise, mobile environmental monitoring station, SMMART.
\end{abstract}

\section{INTRODUCTION}

The growth of the world's population has been followed by the increase of the population living in urban areas, which very often results in additional pressures concerning space, ecosystems, infrastructures, facilities and the way of life [1].

Environmental conditions have an immediate impact on the quality of people's lives [2]. Furthermore, the deterioration of the acoustic and air quality are two of the problems associated with this impact on people's quality of life.

Approximately $20 \%$ of the EU population are exposed to noise levels that are classified as unacceptable by scientists and health experts [3] and at least 1 million healthy life years (disability-adjusted life years) are lost because of environmental noise in western Europe every year [4]. The effect of noise can be physiological, as well as psychological [5]. Moreover, noise leads to annoyance, reduces the quality of the environment and might affect health and cognition [4].

The World Health Organization has recently estimated that ambient pollution is responsible for 3.7 million premature deaths yearly [6]. Among the substances comprising air pollution, Particulate Matter (PM) is seen by some researchers as the most harmful [7, 8]. PM can cause a wide variety of health impacts, such as asthma, bronchitis, cardiovascular diseases and lung cancer [9].

Well-managed urban areas can improve environmental conditions and quality of life [2]. Therefore, evaluating and monitoring the urban environmental quality has become a main issue and is particularly important when it is considered as a decision-making tool that contributes to more habitable and sustainable cities [1]. 
Taking into account these assumptions, the SMMART Vila Real project was developed. SMMART was a project funded by the EU through the platform "AEPLUS", where the evaluation of the urban environment and the divulgation of noise and air pollution levels were considered priority actions. The SMMART project was developed from 2013 to 2014 and the service is available to the public from 2015.

Vila Real is the main city and capital of the district with the same name, situated in the Northeast of Portugal. It has a population of 51,850 inhabitants [10] distributed over an area of $183.4 \mathrm{~km}^{2}$, and about $48 \%$ of the population live in the urban perimeter of the city. In some areas of the world, however, information on air quality is either highly sparse (tending to be located around a particular city or institute) or completely non-existent [11]. This is the case of Vila Real as it does not have any station to evaluate the urban environment within the urban perimeter. The nearest station is located on the Alvão Mountain, located more than 15 kilometres from the city and belongs to IPMA (in English "The Portuguese Institute of Sea and Atmosphere"). Thus, the development of the SMMART project fills this niche.

The SMMART project consists of the following tools: one mobile instrumented unit for environmental monitoring, two long-term noise maps $\left(\mathrm{L}_{\mathrm{den}}\right.$ and $\left.\mathrm{L}_{\mathrm{n}}\right)$ and a long-term $\mathrm{PM}_{10}$ concentration map, traffic statistics from the city's main streets and measurement databases. All this information is available on the following website for public consultation: http:// aeplus.pt/Default

The main focus of this article is a mobile unit developed to monitor the environment in the city of Vila Real from its construction and operation mode of sharing the data.

\section{METHODOLOGY}

In this section, the following will be described: how the unit was constructed from the beginning to the end, the technical characteristics of the equipment and the procedure adopted to collect data for the platform.

\subsection{The mobile unit}

This mobile unit was developed to monitor the environmental parameters at different points of the city and it is an alternative to the fixed stations.

Compared to the fixed stations, the biggest advantage of mobile monitoring is that it can monitor pollutant concentrations at hundreds of spatial points in a region [12]. The costs of setting up fixed site monitoring stations using traditional technology can be substantial. Moreover, individual instruments can cost between $£ 5,000$ and $£ 60,000$ and many other resources are needed for maintenance and calibration [13]. Operating such sites is also limited by the need for significant infrastructure (secure enclosures, mains power, etc.). The consequence is that, while well proven in terms of precision and accuracy of air quality measurements, most existing networks are sparse as higher network densities would be impractical, as well as prohibitively expensive [11]. There is, therefore, an urgent need to complement existing air quality monitoring methodologies with flexible and affordable alternatives, to improve monitoring capabilities for both scientific and legislative purposes, to allow source attribution and to improve understanding of health impacts of urban air quality [11].

Mobile units have some disadvantages when compared to fixed stations. Fixed stations provide a 24-hour a day, 365 days per year measurement record for a given location [14]. However, fixed stations could stop being important for monitoring urban environment, for example, when the location of a station is no longer a strategic point for evaluating urban environments [14]. 
Mobile air pollution monitoring technologies allow for a greater spatial coverage of a city compared to traditional fixed stations [15]. Their limitation is that the monitoring data do not provide a continuous time-series of observations [15]. Mobile units could be used, for example, to respond to complaints about situations where there is a high level of noise or high concentrations of $\mathrm{PM}_{10}$ in a given residential area [14]. Therefore, after analysing the advantages and disadvantages of each station, mobile units were chosen for urban environmental monitoring.

The mobile unit that was chosen is a fully electric car, with a motor of $55 \mathrm{~kW}$ (75 hp) and a battery life of up to 145 kilometers, according to the make. It is a vehicle with small dimensions $(2.69 \times 1.55 \times 1.54 \mathrm{~m})$ and this proved to be a challenge to assemble the working platform with the equipment and the respective accessories.

The mobile unit takes measurements in a specific location of the city (GPS technology), and sends them to a web platform by wireless communication. The following is installed on board the mobile unit: noise modules (type 1 sound level meter), air pollution device (for particle monitoring), a meteorological instrument (weather station), and a communication and positioning device. The equipment is all connected to a router, and communication between the computer and the equipment is made by connecting the computer to the router, i.e. wireless. This communication system, where the devices communicate with the router, has proved to be very efficient because it reduces the number of wires connected to the computer and all the measurement devices (Fig. 1).

\subsubsection{The sliding work platform}

The work platform that was developed is a sliding and robust table installed in the small trunk of the vehicle (capacity of 220 litres up to the cover of the trunk) (Fig. 2). The monitoring devices and respective accessories are distributed over the sliding platform. This structure was designed to support the weight of the devices and to fix them safely to prevent damage while in transit and during operations in the field.

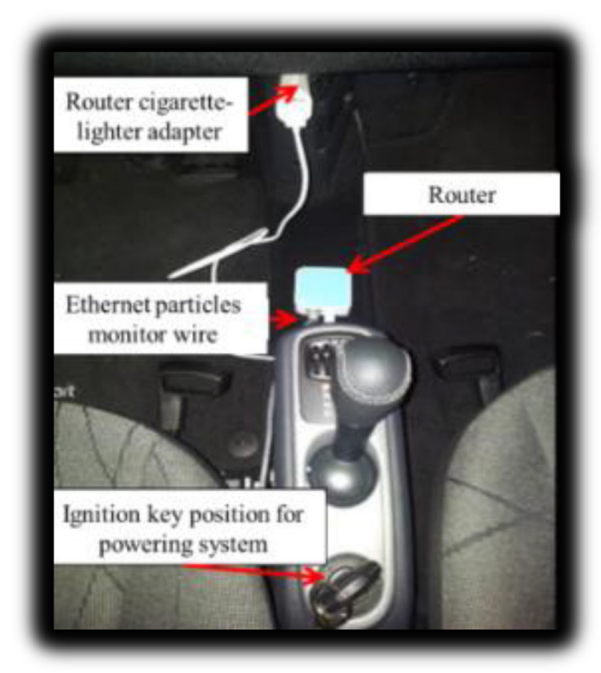

Figure 1: Communication system installed in the vehicle. 


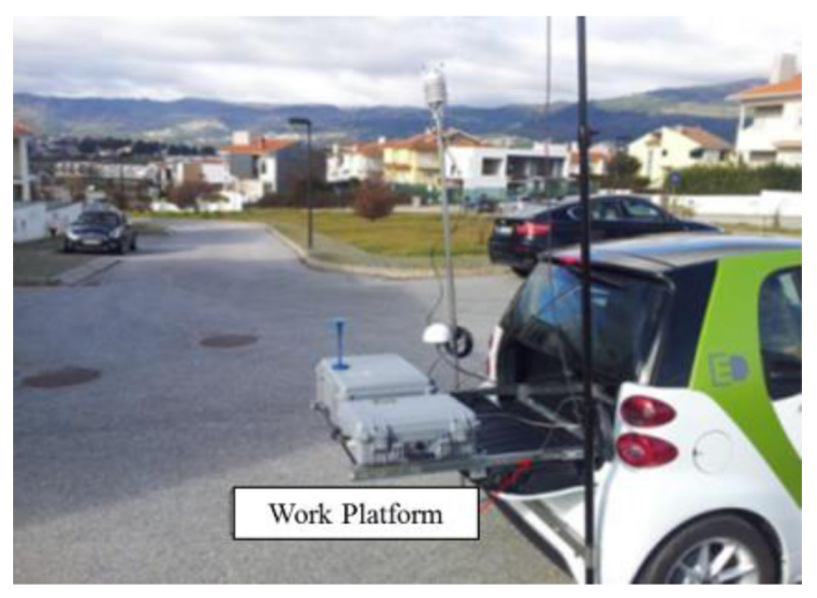

Figure 2: Sliding work platform.

\subsubsection{Device characteristics}

Some technical requirements were taken into account when choosing the devices so that they could be adapted to the vehicle. Weights, dimensions, if they would work outdoors and the battery life were some of the requirements studied.

A PM monitor is a device for measuring real-time aerosol mass readings in the air in an outside environment. This equipment takes measurements of the following pollutants simultaneously: PM10, PM2.5 and Total Suspended Particles (TSP), using a light-scattering laser photometer method. The equipment was installed inside a waterproof enclosure so that accurate measurements could be taken outdoors or in harsh industrial environments. The dimensions, weight and the fact that the equipment is silent (it does not interfere with noise measurements) were other characteristics considered when choosing this equipment. Communication with the computer is made by connecting the Ethernet cable of the device to the router.

A sound level meter is a device which monitors noise in an urban environment. The advantage of using this equipment is the fact that a weather station can be installed to take measurements of noise and meteorological conditions at the same time. The equipment is installed inside a waterproof case. With the sound level meter comes a microphone with an extensible support 4 meters high to take outdoor measurements [16], an external battery for more autonomy, a weather station and a GPS antenna. The sound level meter establishes the connection to the router through Wi-Fi communication.

The final set-up of the monitoring station ready to make measurements is illustrated in Fig. 3.

\subsection{Procedures for collecting data}

The data collection process requires following some procedures. As this is a mobile station for monitoring urban environments, it is essential to take measurements at different points of the city. These points in the city represent the environmental monitoring network of the city. The choice of these points respected the following requisites: 


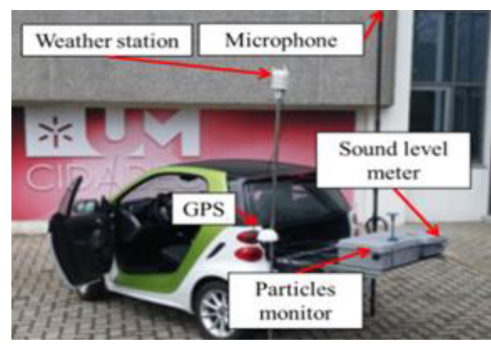

Figure 3: Mobile monitoring station.

- Areas near main roads of the city;

- Places near sensitive areas (schools and hospital);

- Area near the City Park;

- Area near the historical city center.

Therefore, the final monitoring network consists of five sites (Fig. 4): The Câmara Municipal Square (1), Vila Real Hospital (2), Diogo Cão Square (3), Nossa Senhora da Conceição Square (4) and the parking lot near the City Park (5).

After choosing the monitoring points, a plan of action was taken to collect data from five monitoring points in one day of work. Therefore, the total time estimated for the procedure illustrated in Fig. 5 is one hour. This procedure is adopted at every measurement.
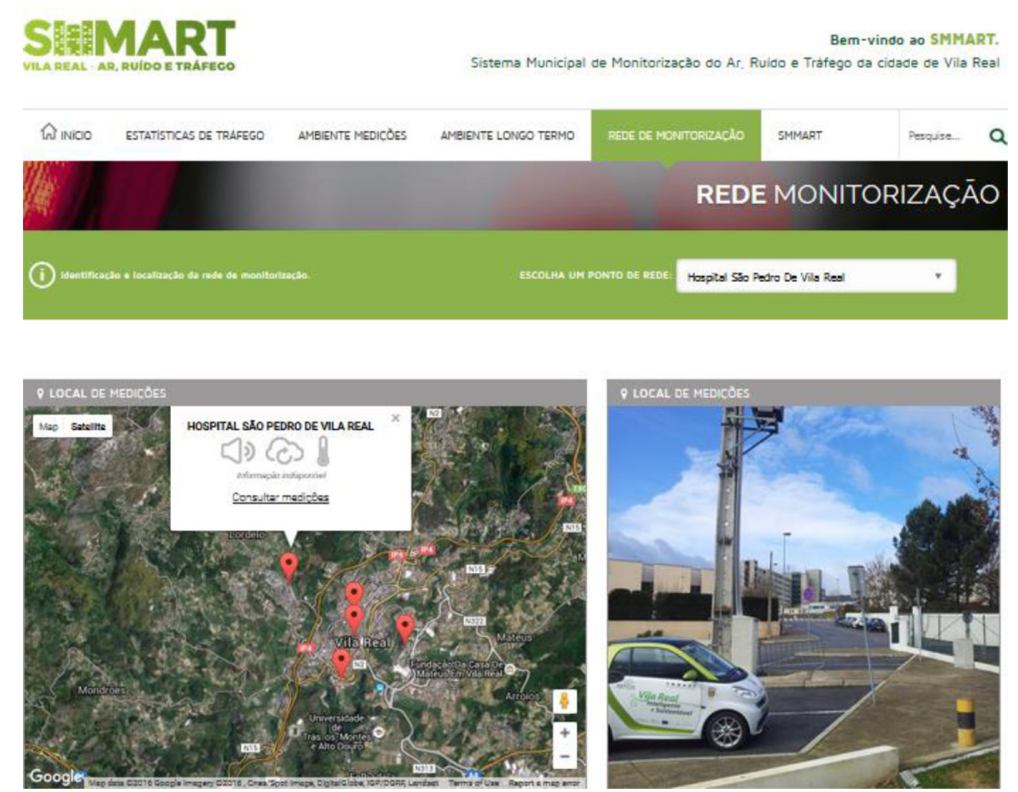

Figure 4: Monitoring network on the SMMART website. 


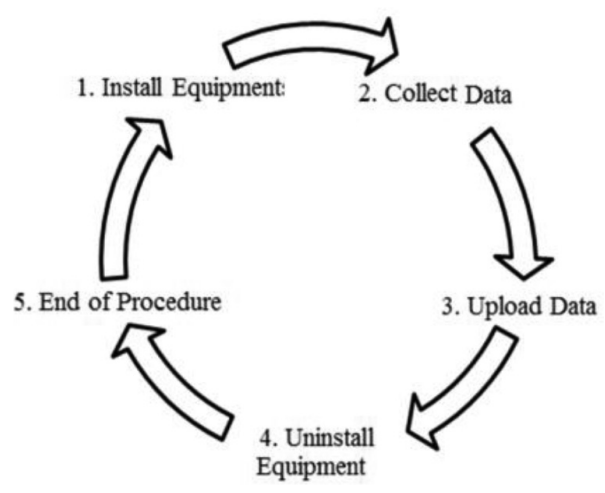

Figure 5: Data collection procedure.

Table 1: Urban pollution indicators.

\begin{tabular}{lllll}
\hline \multicolumn{4}{c}{ Air Quality, Environmental Noise and Heat Index } \\
\hline Very good & Good & Moderate & Poor & Very Poor \\
\hline
\end{tabular}

\subsection{Measurement characteristics}

Every measurement lasts 20 minutes. After assembling the equipment and establishing a connection between the computer and the equipment, the operator, through the computer, gives the order to start taking measurements of all the devices at the same time. After the measurements have been taken, the data is uploaded to the platform, and becomes available for public consultation, after validating the operator responsible for the analysis center.

The file uploading system analyses the data and makes the data available in tabular form. Access to the data is free and can be downloaded by all users in Excel format.

\section{DISCUSSION AND RESULTS}

The results of the measurements can be seen on the following website under "EnvironmentMeasurements": http://aeplus.pt/Default. The results of all the measurements for different monitoring points can be seen individually.

The types of information available to consult are:

- Time- Date and hour of the measurements;

- Noise- $\mathrm{L}_{\mathrm{den}}, \mathrm{L}_{10}$ and $\mathrm{L}_{90}$ (medium values in $\mathrm{dB}(\mathrm{A})$ );

- Particles- PM ${ }_{10}, \mathrm{PM}_{2.5}$ and TSP (medium values in $\mu \mathrm{g} / \mathrm{m}^{3}$ );

- Meteorological parameters- Relative humidity, air temperature and wind speed (medium values) and predominant wind direction (more predominant direction);

Based on the results obtained from the measurements, a classification is given, ranging from very good to very bad, as shown in Table 1 . 
Table 2: Air quality and environmental noise.

\begin{tabular}{lcl}
\hline Air Quality & Environmental Noise & \\
\cline { 1 - 2 } $\mathrm{PM}_{10}\left(\mu \mathrm{g} / \mathrm{m}^{3}\right)$ & $\mathrm{L}_{\mathrm{eq}}(\mathrm{dB}(\mathrm{A}))$ & \\
\hline$[-, 19,9]$ & {$[-, 55]$} & Very Good \\
{$[20,34.9]$} & {$[55,62.5]$} & Good \\
{$[35,49.9]$} & {$[62.5,67.5]$} & Moderate \\
{$[50,119.9]$} & {$[67.5,75]$} & Poor \\
{$[120,+]$} & {$[75,+]$} & Very Poor \\
\hline
\end{tabular}

Table 3: Heat index (values in ${ }^{\circ} \mathrm{C}$ ).

\begin{tabular}{lccccc}
\hline Month & Very Good & Good & Moderate & Poor & Very Poor \\
\hline Jan & {$[18,23]$} & {$[15,18] ;[23,25]$} & {$[13,15] ;[25,28]$} & {$[10,13] ;[28,30]$} & {$[-, 10] ;[30,+]$} \\
Feb & {$[18,23]$} & {$[16,18] ;[22,24]$} & {$[13,16] ;[24,27]$} & {$[11,13] ;[27,29]$} & {$[-, 11] ;[29,+]$} \\
Mar & {$[17,23]$} & {$[15,17] ;[23,25]$} & {$[12,15] ;[25,28]$} & {$[9,12] ;[28,31]$} & {$[-, 9] ;[31,+]$} \\
Apr & {$[17,23]$} & {$[14,17] ;[23,26]$} & {$[11,14] ;[26,29]$} & {$[8,11] ;[29,32]$} & {$[-, 8] ;[32,+]$} \\
May & {$[17,23]$} & {$[13,17] ;[23,27]$} & {$[10,13] ;[27,30]$} & {$[6,10] ;[30,34]$} & {$[-, 6] ;[34,+]$} \\
Jun & {$[22,28]$} & {$[18,22] ;[28,32]$} & {$[15,18] ;[32,35]$} & {$[12,15] ;[35,38]$} & {$[-, 12] ;[38,+]$} \\
July & {$[22,28]$} & {$[18,22] ;[28,32]$} & {$[15,18] ;[32,35]$} & {$[12,15] ;[35,38]$} & {$[-, 12] ;[38,+]$} \\
Aug & {$[22,28]$} & {$[19,22] ;[28,31]$} & {$[16,19] ;[31,34]$} & {$[13,16] ;[34,37]$} & {$[-, 13] ;[37,+]$} \\
Sep & {$[22,28]$} & {$[19,22] ;[28,31]$} & {$[16,19] ;[31,34]$} & {$[13,16] ;[34,37]$} & {$[-, 13] ;[37,+]$} \\
Oct & {$[17,23]$} & {$[15,17] ;[23,25]$} & {$[12,15] ;[25,28]$} & {$[10,12] ;[28,30]$} & {$[-, 10] ;[30,+]$} \\
Nov & {$[17,23]$} & {$[15,17] ;[23,25]$} & {$[12,15] ;[25,28]$} & {$[10,12] ;[28,30]$} & {$[-, 10] ;[30,+]$} \\
Dec & {$[18,22]$} & {$[15,18] ;[22,25]$} & {$[13,15] ;[25,27]$} & {$[10,13] ;[27,30]$} & {$[-, 10] ;[30,+]$} \\
\hline
\end{tabular}

This classification is given individually to each environmental indicator (environmental noise, air quality and heat index) using multi-criteria developed algorithms, which combines the measurement values with the tables below [1]. The ranges of values given to the classifications are explained in Table 2 (environmental noise and air quality) and Table 3 (heat index):

The classification illustrated in Table 3 was based on mean temperatures and respective standard deviations registered in the city of Vila real over the last 50 years [17].

On the website (http://aeplus.pt/Default), other information can be consulted. Among this information is: traffic statistics, two long-term noise maps $\left(\mathrm{L}_{\mathrm{den}}\right.$ and $\left.\mathrm{L}_{\mathrm{n}}\right)$, an atmospheric pollution map $\left(\mathrm{PM}_{10}\right)$ and an area especially for public awareness.

The traffic statistics were obtained from $24 \mathrm{~h}$ vehicle counting, resources (videos) and a device that counts the number of vehicles in the main streets of the city. Counting took place on week days (except Saturdays and Sundays) in March and April, 2014. The traffic was separated into vehicle type: light vehicles, motorcycles and heavy vehicles. As well as traffic counts, other information could be consulted, for example information about the road structure (number of traffic lanes, street orientation and number of traffic directions). 
Long-term noise maps $\left(\mathrm{L}_{\mathrm{den}}\right.$ and $\left.\mathrm{L}_{\mathrm{n}}\right)$ and an air pollution map $\left(\mathrm{PM}_{10}\right)$ were also included in the platform (Fig. 6). The noise and air pollution sources considered for devising the maps were the road traffic. A mobile unit was used to validate the maps by measuring different points in the city (monitoring network and other points), by comparing the modulated values, as well as the result values from the measurements. To consider the noise maps to be valid, the difference between the modeled and measured values must be less than $\pm 3 \mathrm{~dB}(\mathrm{~A})$.

The final item on the platform is an area for Public Awareness. This was designed to inform the population about possible abnormal conditions that may influence the air and noise quality (a text). For example, high levels of noise for a given zone in the city, warnings about high levels of particle concentrations that can affect the health of high risk age groups, warnings about road works and road accidents are some kinds of information which can be consulted in the Public Information area.

\section{CONCLUSIONS}

Monitoring and informing the population about the quality of the urban environment using a monitoring mobile unit was the main focus of this article.

In this paper, all the stages of the project were presented from constructing the mobile monitoring unit with all the measurement devices to data collection procedures, setting up the monitoring network and following procedures to classify the urban environment.

The mobile unit has shown that it can develop the tasks for which they were developed. The validation of long-term noise maps and $\mathrm{PM}_{10}$ concentration map, as well as in regular measurements for environmental monitoring, was successfully accomplished with the use of the mobile unit.
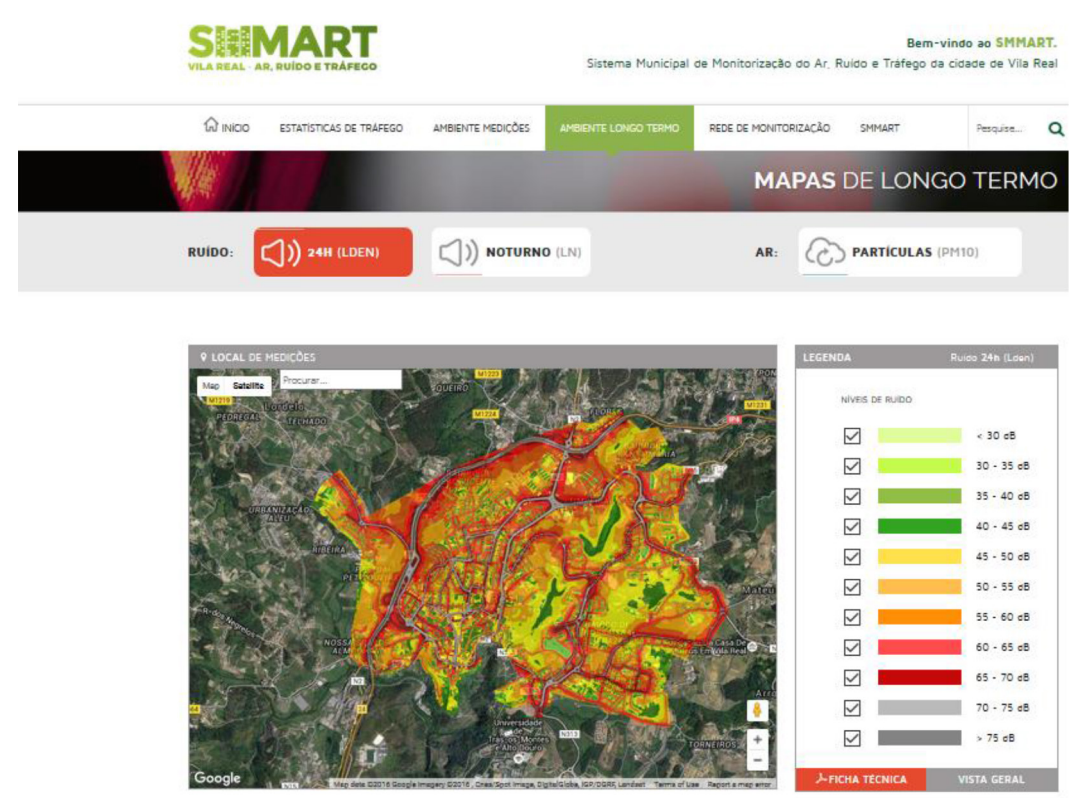

Figure 6: Long term-maps on the SMMART website. 
Despite the less accuracy in the data collection when compared to fixed stations, the mobile unit has proved to be capable of monitoring the environment efficiently at different points of the city.

Finally, the instrumented mobile unit has shown to be efficient and well accepted by the municipality and population, providing credible information about urban environmental parameters and reflecting the existing reality.

\section{ACKNOWLEDGEMENTS}

The authors would like to thank the technical team from the municipality of Vila Real for all the available information, knowledge of the territory and support, as well as the essential requirements to develop this research. Additionally, the authors would like to thank MRA for the technical support in installing the sliding platform in the mobile monitoring unit.

\section{REFERENCES}

[1] Mendes, J.F.G., Silva, L.T., Ribeiro, P. \& Magalhães, A., An urban environmental monitoring and information system. Air Pollution, XVII, 2008.

[2] Silva, L.T., Environmental quality health index for cities. Habitat International, 45, 2015.

[3] CALM II Network, Research for a Quieter Europe in 2020. An Updated Strategy Paper of the CALM II Network. R. D.-G. European Comission, Brussels, 2007.

[4] WHO, Burden of Disease from Environmental Noise, World Health Organization: Bonn, 2011.

[5] Muzet, A., Environmental noise, sleep and health. Sleep Medicines Review, 11, pp. 135-142, 2007.

http://dx.doi.org/10.1016/j.smrv.2006.09.001

[6] WHO, Frequently asked questions: ambient and household air pollution and health, available at http://www.who.int/phe/health_topics/outdoorair/databases/faqs_air_pollution.pdf?ua=1 (accessed 28 October 15), 2014.

[7] Cohen, A.J., Ross Anderson, H., Ostro, B., Pandey, K.D., Krzyzanowski, M., Künzli, N., Gutschmidt, K., Pope, A., Romieu, I., Samet, J.M. \& Smith, K., The global burden of disease due to outdoor air pollution. Journal of Toxicology and Environmental Health. Part A, 68, pp. 1301-1307, 2005.

http://dx.doi.org/10.1080/15287390590936166

[8] European Environment Agency, Air Quality in Europe 2013 Report, EEA report, Copenhagen, available at http://bookshop.europa.eu/en/air-quality-in-europepbTHAL1301 0/?CatalogCategoryID=h2YKABstrXcAAAEjXJEY4e5L (accessed September 2015).

[9] Anderson, J.O., Thundiyil, J.G. \& Stolbach, A., Clearing the air: a review of the effects of particulate matter air pollution on human health. Journal of Medical Toxicology, $\mathbf{8}$, 166-175, 2012.

http://dx.doi.org/10.1007/s13181-011-0203-1

[10] Censos 2011, Instituto Nacional de Estatística (INE), Portugal, 2011.

[11] Mead, M.I., Popoola, O.A.M., Stewart, G.B., Landshoff, P., Calleja, M., Hayes, M., Baldovi, J.J., Hodgson, T.F., McLeod, M.W., Dicks, J., Lewis, A., Cohen, J., Baron, R., Saffell, J.R. \& Jones, R.L., The use of electrochemical sensors for monitoring urban air quality in low-cost, high-density networks. Atmospheric Environment, 70, pp. 186-203, 2013.

http://dx.doi.org/10.1016/j.atmosenv.2012.11.060 
[12] Su, J.G., Hopke, P.K., Tian, Y., Baldwin, N., Thurston, S.W., Evans K. \& Rich, D.K., Modeling particulate matter concentrations measured through mobile monitoring in a deletion/substitution/addition approach. Atmospheric Environment, 122, pp. 477-483, 2015.

http://dx.doi.org/10.1016/j.atmosenv.2015.10.002

[13] Ropkins, K. \& Colvile, R.N., Critical review of air quality monitoring technologies for urban traffic management and control (UTMC) systems. Urban Traffic Management \& Control (UK), 2000.

[14] Butterwick, L., Harrisons, R. \& Merritt, Q., Handbook for Urban Air Improvement. Commission of the European Communities, 1991.

[15] Adams, M.D. \& Kanaroglou, P.S., Mapping real-time air pollution health risk for environmental management: Combining mobile and stationary air pollution monitoring with neural network models. Journal of Environmental Management, 168, pp. 133-141, 2016.

http://dx.doi.org/10.1016/j.jenvman.2015.12.012

[16] European Directive 2002/49/EC of the European Parliament and of the Council of 25st of June 2002.

[17] Heat Index Calculator, Weather Prediction Center, National Weather Service, USA. 